\title{
Uma investigação sobre automutilação em um grupo da rede social virtual Facebook*
}

Aline Conceição Silva ${ }^{1}$

Nadja Cristiane Lappann Botti

Com o objetivo de identificar a composição e estrutura de um grupo de automutilação em rede social virtual foi realizada pesquisa qualitativa, descritiva e exploratória. Foram analisadas 103 postagens textuais e imagéticas, a partir da análise de conteúdo de Bardin, de um grupo sobre automutilação no Facebook no período de 2014-2015. As categorias, composição e estrutura do grupo de automutilação no Facebook, que emergiram abordam características identitárias que permeiam os participantes do grupo e contexto da prática de automutilação e atitudes de engajamento ao grupo. Ressalta-se que as características identitárias e estruturais encontradas que operacionalizam a funcionalidade de um grupo de automutilação na internet colaboram para a compreensão do comportamento e contribuem para prática profissional infanto-juvenil.

Descritores: Automutilação; Adolescente; Internet.

\footnotetext{
* Artigo extraído de dissertação de mestrado "Comportamento autolesivo não suicida em rede social virtual" apresentada à Universidade Federal de São João del-Rei, Divinópolis, MG, Brasil.

${ }^{1}$ MSc, Enfermeira.

${ }^{2} \mathrm{PhD}$, Professor Adjunto, Universidade Federal de São João del-Rei, Divinópolis, MG, Brasil.
} 


\section{An investigation on self-mutilation in a group of the social network Facebook}

To identify the composition and structure of a self-mutilation group in a virtual social network, qualitative, descriptive and exploratory research was carried out. We analyzed 103 textual and imagery posts, based on the content analysis of Bardin, from a group about self-mutilation on Facebook in the period 2014-2015. The categories, composition, and structure of the Facebook self-mutilation group that emerged address identity characteristics that permeate group participants and the context of self-mutilation practice and engagement attitudes to the group. It is emphasized that the identity and structural characteristics found that operationalize the functionality of a self-mutilation group on the internet contribute to the understanding of the behavior and contribute to the professional practice of children and youth.

Descriptors: Self Mutilation; Adolescent; Internet.

\section{Una investigación de la automutilación en un grupo virtual de red social}

\section{Facebook}

Con el objetivo de identificar la composición y estructura de un grupo de automutilación en red social virtual se realizó una investigación cualitativa, descriptiva y exploratoria. Se analizaron 103 posturas textuales e imágenes, a partir del análisis de contenido de Bardin, de un grupo sobre automutilación en Facebook en el período de 2014-2015. Las categorías, composición y estructura del grupo de automutilación en Facebook, que emergieron abordan características identitarias que permean a los participantes del grupo y contexto de la práctica de automutilación y actitudes de compromiso al grupo. Se resalta que las características identitarias y estructurales encontradas que operan la funcionalidad de un grupo de automutilación en Internet colaboran para la comprensión del comportamiento y contribuyen a la práctica profesional infanto-juvenil.

Descriptores: Automutilación; Adolescente; Internet.

\section{Introdução}

Automutilação é definida como comportamento intencional de destruição ao próprio corpo e sem intenção suicida $^{(1)}$. A adolescência é o período predominante para o comportamento(2), sendo mais frequente em menina que meninos, $17 \%$ e $7,9 \%$, respectivamente ${ }^{(3)}$. Estima-se que a prevalência de automutilação no Reino Unido situa-se entre $4,6 \%$ e $6,6 \%{ }^{(3)}$. No Brasil ainda não existem estudos que quantifiquem a prevalência deste tipo de comportamento. Entretanto evidenciase o comportamento autolesivo como um fenômeno oculto, ou seja, o número de casos de automutilação que comparece aos serviços de saúde é muito pequeno perto dos casos sem tratamento na comunidade ${ }^{(4)}$.

A Internet possui impacto potencial no desenvolvimento de comportamento autolesivo não suicida e suicídio, neste contexto, pode se apresentar como ambiente de risco colaborando com a efetivação do comportamento, como também pode operar como estratégia contemporânea de prevenção. Atualmente a Internet faz parte do cotidiano de milhares de pessoas em todo o mundo, com características como atemporalidade e extraterritorialidade vêm ganhando cada vez mais adeptos. No Brasil cerca de $76 \%$ das pessoas acessam a Internet todos os dias com exposição média diária 
de 4 h59 de segunda a sexta(5). No Reino Unido, mais de $80 \%$ dos domicílios tem acesso à Internet ${ }^{(6)}$. No mundo, mais de 700 milhões de pessoas possuem perfis online em Redes Sociais Virtuais, tornando-se um meio moderno e primário de interação social ${ }^{(7)}$.

É notório que a Internet atua como um importante recurso de conectividade entre os indivíduos, principalmente indivíduos que se sentem isolados. Estudos apontam que a Internet intervém sobre indivíduos vulneráveis de duas formas principais: como espaço de apoio mútuo, com troca de experiências e suporte emocional, diminuindo sentimentos de solidão e isolamento, ou como espaço nocivo com troca de informações anônimas ou com gatilhos para comportamentos de risco(8).

A propagação e alcance da Internet tem chamado a atenção do público em geral, especialmente pais de crianças e adolescentes, além de profissionais da área da saúde e dos governos em relação ao problema do suicídio e da automutilação( ${ }^{(9)}$. Estudo realizado na Itália mostra grande interesse na busca sobre automutilação na internet, principalmente com os termos "autolesão e corte" seguido de "autolesão e filho" "autolesão e fóruns" e "autolesão e causas e razões"(10). Estudo irlandês realizado com 3500 escolares aponta que 18\% que haviam se auto lesionado indicaram a influência da internet na sua decisão de envolver-se com a automutilação ${ }^{(11)}$. Ainternet tem levado a criação de novos espaços virtual e social na vida das pessoas, oferecendoas novas perspectivas na vivência de conhecimentos bons e ruins ${ }^{(12)}$. Sabe-se que da inovação de uma nova tipologia de comunicação social através da publicação de imagens e elementos textuais no ambiente online. Mas apesar de sua grande publicidade, ainda pouco se tem estudado sobre sua influencia na vida do ser humano ${ }^{(13)}$. A fim de estudar o comportamento da automutilação no contexto brasileiro foi realizada uma investigação objetivando identificar a composição e estrutura de um grupo de automutilação da Rede Social Virtual.

\section{Método}

O estudo teve uma abordagem qualitativa, descritiva e exploratória, fundamentada pelo referencial teórico da análise de conteúdo de $\operatorname{Bardin}^{(14)}$. De acordo com este referencial a análise das comunicações permite a "inferência de conhecimentos relativos às condições de produção e recepção de mensagens"(14). Buscou-se por intermédio dessa pesquisa, apresentar os significados manifestados nas mensagens das postagens e comentários de um grupo de automutilação da Rede Social Virtual (RSV) Facebook.
A pesquisa foi realizada num grupo sobre automutilação encontrado a partir da barra de busca da Rede Social Virtual Facebook com o termo "automutilação". O grupo opera desde 2013 e tem se classifica como grupo de apoio. O grupo foi escolhido por possuir como objetivo ajudar os praticantes de automutilação na troca de informações, funcionar como grupo de apoio para pessoas vulneráveis ao comportamento autolesivo e ter grande número de participantes (7.471 participantes).

O Facebook categoriza os grupos como grupo aberto, fechado ou secreto. A principal diferença dos três está na permissão de visualizar as postagens, sendo o grupo aberto o único que permite a visualização sem participar do grupo. Os grupos fechados e secretos precisam de permissão para participar e consequentemente visualizar as publicações dos participantes. $\mathrm{O}$ grupo deste estudo enquadra-se na categoria grupo fechado, sendo necessária a aprovação das pesquisadoras por administradores da página. A aprovação das autoras não esteve condicionada a questionamentos ou motivação para participação. A pesquisa não foi divulgada para os participantes e/ou administradores e foi requisitado ao Comitê de Ética e Pesquisa com Seres Humanos da Universidade Federal de São João del-Rei (CEPS/UFSJ) a dispensa do Termo de Consentimento Livre e Esclarecido. Estas decisões foram adotadas em conjunto com o CEPES/ UFSJ devido ao extenso número de participantes e que a divulgação da pesquisa poderia fragilizar os laços entre pesquisador e pesquisado. $O$ estudo está em consonância com os objetivos e pressupostos da Resolução 466 de dezembro de 2012.

Os dados foram coletados de março a abril de 2016, após a aprovação do projeto dessa pesquisa pelo CEPES/UFSJ, com parecer número 975.511, em 19 de março de 2015. A coleta dos dados foi realizada no grupo de automutilação da Rede Social Virtual Facebook no por meio das postagens com mais de 10 comentários e comentários dos mesmos publicados no grupo no período de dezembro de 2014 a dezembro de 2015. Para a coleta foi utilizado formulário elaborado pelas autoras e construído no programa Microsoft Word 2010. O formulário contemplava questões como: data e horário da postagem, se postagem principal (texto) ou secundário (foto ou link em anexo) e por fim comentários e números de curtidas. Eles foram identificados atribuindo-se a letra $\mathrm{P}$ para as postagens e a letra $C$ para os comentários.

Os dados foram analisados segundo a proposta operativa de Bardin ${ }^{(14)}$, a qual recomenda uma préanálise a partir da leitura flutuante do material coletado, definição das unidades de registro e significação com a 
construção das categorias de análise e posteriormente inferência e interpretação dos resultados à luz do referencial teórico. Como o objetivo do estudo referese identificar a composição e estrutura do grupo de automutilação da Rede Social Virtual Facebook foi utilizado para análise das postagens e comentários um roteiro norteador contendo duas questões: 1 - Quem são os participantes do grupo de automutilação da Rede Social Virtual Facebook?; 2- Como o grupo de automutilação da Rede Social Virtual Facebook encontra-se estruturado?

Após leitura do material, foram identificadas as principais unidades de registros que estavam presentes na maioria dos discursos e categorizadas por similaridades. A codificação foi realizada primariamente por uma das autoras do estudo sendo discutida posteriormente com professora Doutora em Enfermagem Psiquiátrica pela Universidade de São Paulo e com ampla experiência em pesquisas com o referencial metodológico adotado.

\section{Resultados}

A partir da análise dos depoimentos e comentários publicados no grupo virtual de automutilação emergiram duas categorias: composição e estrutura do grupo de automutilação da Rede Social Virtual Facebook.

\section{Composição do grupo de automutilação da Rede Social Virtual Facebook}

O conteúdo dessa categoria refere-se à composição de características identitárias que permeiam os participantes do grupo e que se apresentam como: identificação relacionada com a faixa etária, sintomas contemporâneos, ídolo juvenil e com o tipo de comportamento específico de automutilação. Os depoimentos e comentários dos participantes do grupo revelam participantes jovens e a iniciação da prática da automutilação na adolescência: Comecei com 15 anos (tenho 17)... (C2028). Tenho 15 anos e comecei aos meus 11 anos... (C2033). Eu acho que sou a mais nova do grupo [emoticon frown] (carinha triste) eu tenho 11 anos (C2034). Tenho 17, comecei aos 13 (C2036). Tenho 19, me corto desde que tinha 10 anos (C2037). Eu tenho 14 anos e comecei com 11 anos :"/ (C2043). Tenho 13 e comecei esse ano ah uns 7 meses atrás (C2056).

Observa-se a associação do comportamento de se automutilar com sintomas contemporâneos dos transtornos alimentares pelos depoimentos e comentários pró-anorexia e bulimia. Importante ressaltar que os participantes do grupo identificam os transtornos alimentares através dos termos ana e mia, ou seja, anorexia e bulimia respectivamente. Alguém aqui é próana? (P11). Gente serio mesmo eu sou A\&M (C1012). Alguém aqui pratica ana e mia? (P65). Eu tenho um grupo no whats pra ana e mia Se alguém quiser entrar me manda o número inbox (C2166). Minhas rainhas ana e mia (C2165). Eu sou Mia e pró-ana (C151).

De acordo com os depoimentos e comentários dos participantes observa-se identificação do grupo com a cantora e compositora norte-americana Demi Lovato que apresenta história de automutilação e transtornos alimentares. Os participantes do grupo de automutilação se identificam como Lovatics e referenciam a expressão Stay Strong, termo tatuado pela cantora em seus pulsos para representar o apoio dos fãs durante o tratamento da automutilação. Importante frisar que após o tratamento a cantora dedicou-se a ofertar mensagens de apoio aos fãs que sofrem com o comportamento autolesivo. Vc também é lovatic? E foca nisso, você é como a Demi pode vencer!!! Você já é forte de aguentar 3 meses. Continue assim! Não desista! Estamos torcendo por você!!! (C214). Eu fiquei 1 mês sem cortes e ontem me cortei achando q tbm eu ia melhorar. Mas isso $n$ vai me fazer desistir, Nós merecemos ser felizes! Stay Strong. (C104).

Identifica-se no grupo a presença de automutilação grave como vários cortes ou queimaduras na pele e o interesse por informações sobre objetos cortantes, extensão de cortes e cicatrizes: Com o que vocês se cortam? Eu me corto com as lâminas da gillete mas não são tão fundos, queria que fossem (P52); Me cortava com a tesoura, e lâminas de apontador... ficaram beeem fundos (C1010). Eu me cortava com filete tesouras e me queimava mas consegui sair, igual VC um dia vai (C1011). Sei que esse não é o tipo de conselho que eu deveria das, MAS eu usava a lamina da gilete mesmo, quem determina a profundidade ou a marca que vai ficar é você mesma. As minhas estão aqui a anos (C1023). Tem pessoas q não se controlam, pra não usar moletom, cortava as coxas e barriga... os pulsos eu cobria com pulseiras, quem se automutila, possui essa necessidade (C965). Não queria cortes fundos, eu sei que é isso e pode ter certeza não é legal... Todos olham, reparam perguntam e da um constrangimento danado. Se vc tem cortes fundos é o que mais vai chamar atenção, vão achar que vc é louca porque ninguém entende seus motivos na verdade... E pior o maior julgamento é da sua própria família (C1026).

Estrutura do grupo de automutilação da Rede Social Virtual Facebook

Esta categoria apresenta as estruturações realizadas pelos próprios participantes do grupo de 
automutilação em rede social virtual para operacionalizar a funcionalidade do grupo. Assim, compreende como estruturas do grupo online os significados e as motivações para a prática da automutilação evidenciando o contexto da prática da automutilação entre os participantes do grupo e atitudes acolhedoras e de compreensão contribuindo no engajamento de participantes ao grupo.

Entre os significados da prática de automutilação encontra-se a expressão de um sofrimento e manifestação patológica com característica de comportamento dependente. A automutilação é uma doença! Precisa de cuidados. A automutilação não é legal, não é normal, e não tem a menor graça! Nunca me orgulhri dos meus cortes! Nunca gostei de me cortar! (C740). E cada dia piora o vicio (C2029). Se cortar uma única vez?? A maioria dos vícios começa com a pessoa experimentando (C652). Não estamos falando de um cortinho estamos falando de pessoas que se mutilam e isso é grave (C739). Pensei que voltando para as lâminas as coisas iam mudar, os sentimentos iam sumir, mas tudo ficou do mesmo jeito, os cortes em meus pulsos doem (P07). Corte pra mim, é sofrimento! Afff (C1618). Só a lâmina realmente me entende! E eu pensei... Por alguns dias, que estava curado! Mas com o tempo tudo volta, aí o tempo fica turvo e a única coisa que se enxerga é a lâmina : / : :/ (P96). Eu me cortei durante 3 anos... Eu queria parar, mas acabava sendo "automático".. às vezes, eu me cortava $p q$ alguém falava um pouco mais alto comigo. Não me cortava pq eu queria, e sim pq eu estava sensível. Eu queria parar, mas não tinha quem me ajudasse. Sim! O [grupo] fez um papel muito importante nessa minha recuperação (C572). Tanto tempo sem se cortar... mais tive uma recaída a alguns dias, infelizmente! Emoticon unsure (carinha insegura) (P89)

A categoria motivações para a prática da automutilação apresenta as causas apresentadas pelos participantes do grupo do Facebook. Neste sentido observa-se que as causas têm relação com a fuga de problemas; psicopatologias, busca de atenção; desamparo; falta ausência ou omissão dos pais e sensação provocada pelo próprio ato: O meu primeiro corte foi com 9, mas eu n fiz mais. Ai começaram aparecer muitos problemas na minha vida que eu fui fazendo mais, mais, mais e mais (C2053). Eu estava a um ano tão feliz com toda essa mudança e tudo voltou outra vez, a angústia, tristeza, as vezes me sinto abandonada... (P89). Nem eu sei como lidar com isso... eu tenho muitos pesadelos com violência sexual... Sinto nojo do meu corpo... as vezes não suporto me tocar... me olhar... e difícil... (C472). Acabei de ser traída enquanto estava em um relacionamento sério (P61). É complicado. As vzs eu faço de raiva, frustações cotidianas e depressão... (C2050). Eu me cortava pq eu realmente tinha motivos eu me cortava pq eu sou bipolar eu tinha depressão e outras coisas mais. (C659). Muitas vezes os filhos só querem ser melhores para os pais só querem que os pais tenham orgulho deles.... Se tivéssemos mais pais presentes muitas pessoas não começariam a se cortar eu tenho certeza (C1909). Comecei a me cortar depois q meu pai morreu a 3 anos e meio, e eu queria realmente morrer, nunca quiz tanto pois quando meu velho morreu eu estava brigada com ele pelo fato dele usar drogas nunca estar em casa e não se importar comigo. Quando soube que ele foi assassinado na cadeia eu quiz ser ele. Porque ele teve que morrer? (P88).

A subcategoria de atitudes acolhedoras e de compreensão é evidenciada em muitos comentários e demonstra aspectos de inclusão e engajamento no grupo de automutilação em redes sociais virtuais: Nesse grupo, tem pessoas maravilhosas... Que realmente querem ajudar. Não estou falando isso pra puxar saco de ninguém não... Estou falando pq eu sei que são, pois quando precisei, elas me ajudaram de verdade. Não custa nada chamar uma de nós pra conversar (C585). Quantas vezes eu conversei com alguém que evitou que eu me cortasse... (C587). Aqui vc pode ajudar ou ser ajudada caso precise de ajuda... Emoticon grin (carinha sorrindo), estamos aqui para o q precisar (C1578). Eu me cortava passei dois anos me cortando faz duas semanas que não me corto e estou aqui pra ajudar que precisa (C1581). Mas o grupo não eh pra quem gosta de se cortar e sim pra qm quer parar se de se machucar (C1621)

Entretanto os depoimentos e comentários dos participantes apresentam divergências quanto às regras e entendimento do que é fator protetivo e de risco para automutilação. Identifica-se a preocupação entre os participantes quanto à publicação de fotos de cortes como fator de risco para o comportamento de se automutilar: Este é um grupo que foi criado com o propósito de ajudar-nos uns aos outros a superar o problema que é a automutilação. Está explícito que a maioria aqui não se sente bem, então acho desnecessário postar fotos que muitas vezes atrapalham o processo de recuperação e superação de outra pessoa. Está se sentindo mal? Conte-nos a sua história e tentaremos de coração ajudar, apenas isso basta. (C581). Não é egoísmo, porque não postam foto pensando "a vou postar pra outra pessoa ver e se cortar", mas porque querem ajuda, só que as vezes afeta alguém, acredito que a maioria das pessoas que postam foto aqui é para pedir ajuda... nem sempre tem coragem de chamar alguém assim do nada e falar o que esta acontecendo" (C586). Nossa, o grupo é pra ajuda e o maior foco é a Automutilação. Se as pessoas postam fotos dos cortes aqui é porque sabem que aqui encontraram a melhor ajuda, e não julgamento. Postam para ver se alguém se importa de verdade e assim oferece ajuda, não querem alguém que sintam a obrigação de ajudar, e sim alguém que ofereça essa ajuda por livre e espontânea vontade, assim, sentem que alguém realmente se importa (C777). 


\section{Discussão}

A adolescência é um período de maturação que sofre influências sociais, culturais e ambientais com exposição a diferentes situações de vulnerabilidade à sua saúde ${ }^{(15)}$. Nesta etapa do ciclo vital a afiliação a grupos sociais encontra-se associada a níveis mais baixos de vulnerabilidades e comportamentos autodestrutivos $^{(16)}$. O comportamento autolesivo pode acontecer em qualquer etapa do ciclo vital, entretanto, estudos apontam a adolescência como período predominante para o comportamento(2). ${ }^{(2)}$ comportamento autolesivo inicia-se geralmente aos 13, 14 anos, podendo perdurar por 10 a 15 anos, ou mesmo por décadas e comumente, encontrase associado ao mecanismo de enfrentamento mal adaptado característico da adolescência(1).

Os transtornos alimentares configuram-se como sintomas contemporâneos associados a representações e significados que fornecemos a ingesta do alimento agregado aos valores e padrões impostos pela sociedade atual ${ }^{(17)}$. Os transtornos alimentares (anorexia e bulimia) estão associados a graves distúrbios de saúde, entre eles a automutilação, refletindo dificuldades mais amplas nos mecanismos eficazes de enfrentamento(18).

O comportamento autolesivo latente prediz significativamente a aversão ao tamanho do corpo indicando que pessoas com história de automutilação são menos sensíveis ao tratamento. Ainda o comportamento autolesivo é importante fator prognóstico para os transtornos alimentares em função de sua associação com experiências de efeito negativo e sintomas de ansiedade e depressão que por sua vez favorecem a insatisfação corporal após o tratamento dos transtornos alimentares ${ }^{(18)}$.

No estudo(17) sobre transtornos alimentares e comunidades virtuais, as autoras encontraram dados que corroboram com os achados do presente estudo: comunicação própria, por exemplo, ana (anorexia), mia (bulimia), identificação com pares, sensação de pertencimento, identificação e influência de ídolos (atrizes, cantoras, modelos) presença característica em comunidades virtuais pró ana e mia e que servem de estímulo para esse comportamento.

A adolescência contemporânea é marcada pela "solidão afetiva", com a ausência dos pais e a introdução de cuidadores pagos, associados a computadores, televisores e vídeo games de última geração( ${ }^{(19)}$. Dessa forma as referências desses adolescentes não são mais os pais ou outros cuidadores familiares, mas sim personalidades impessoais de telenovelas, filmes, ícones da música, moda e esportistas que serão os modelos identificatórios e que servirão de "eixo valorativo para a construção do estilo de vida" dos adolescentes ${ }^{(19)}$.

O seguimento de pessoas que exercem influência entre adolescentes expandiu enormemente depois do advento das mídias de comunicação em massa ${ }^{(20)}$. O estudo(21) com adultos jovens evidencia que $75 \%$ destes relataram forte atração por uma celebridade em algum momento de suas vidas, principalmente músicos ou astros do cinema. Ainda, que $59 \%$ dos entrevistados sentiram-se influenciados por seus ídolos em algum aspecto de suas crenças ou atitudes. Neste aspecto entende-se que adolescentes são influenciáveis a comportamentos de celebridades que podem tornarse referências para o início e perpetuação de atos que coincidam com a realidade vivenciada pelos mesmos ${ }^{(22)}$.

Encontra-se como característica identitária dos participantes do grupo o tipo específico do comportamento de se automutilar relacionado com particularidades do auto corte. Segundo a Escala de Comportamento de Automutilação (Functional Assessment of Self-MutilationFASM) traduzida e adaptada para o português permite caracterizar a gravidade da lesão(1).

A adesão em grupos sociais é uma característica fundamental da existência humana ${ }^{(23)}$ que em geral envolve atividades relacionadas a sentimentos de interação, pertença, filiação e conexão entre os participantes. Tais sentimentos são entendidos como positivos para a identificação social e importante característica da adolescência ${ }^{(16)}$.

Destacam-se três características importantes ao engajamento em grupos sociais relacionados à identidade social: os participantes de grupos tendem a cooperar e ajudar outras pessoas com as quais se identificam; são susceptíveis a receber tais benefícios de outros membros; e identificação com grupos sociais (família, escola, amigos) relaciona-se a laços de apoio e, portanto, com níveis elevados de bem-estar psicológico e baixos de sintomas psiquiátricos auto relatados ${ }^{(16)}$.

Atualmente, os grupos sociais se expandem cotidianamente através da Internet, na qual pessoas com ensejos, sofrimentos ou outras peculiaridades similares podem trocar informações e se aconselharem sem adscrição de localidade ou temporalidade. Dessa forma os benefícios da inserção em grupos sociais estendemse também para os grupos sociais online (ou virtuais) caracterizados pelos depoimentos nas postagens do grupo de automutilação em Rede Social Virtual.

A partir do pressuposto de comportamento dependente $^{(24)}$, compreende-se os conceitos de recaída e lapso. Entende-se recaída como um retorno à mesma intensidade do comportamento dependente, por exemplo, uma série de automutilações, enquanto que um lapso pode ser descrito como o corte inicial depois de um 
período sem o ato ou um evento discreto, diferente da recaída, que envolve neste exemplo a série de automutilações continuadas depois deste deslize inicial.

Importante frisar que a exposição a conteúdos sobre automutilação pode envolver contágio social, sendo um gatilho para este tipo de comportamento. $\mathrm{O}$ contágio social pode ser off-line ou online, no último caso ainda se tem a criação de perfis anônimos e a exposição a grande quantidade de perfis e troca de informações. A natureza visual deste tipo de comportamento é de fácil acesso e altamente disponível, podendo ser potencialmente imitado(22).

Entre os motivos para o comportamento autolesivo seria requerer ajuda ou suporte além de exercer influência interpessoal(22). Um dos pontos cruciais ao se participar de grupos sociais virtuais é a busca de auxílio por identificação com pessoas que estão na mesma situação, assim, se os participantes envolvidos em tais grupos têm boas intenções ou resposta terapêutica ao sofrimento pode-se creditar que o apoio social terá efeito positivo sobre o comportamento(2).

Entre os significados da prática de automutilação encontra-se a expressão de um sofrimento e manifestação patológica com característica de comportamento dependente. Os comportamentos dependentes são transtornos complexos que se multiplicam a partir de predisposição genética, processos socioculturais, vulnerabilidades psicológicas, expectativas cognitivas positivas sobre os efeitos e consequências do consumo de álcool e outras drogas (bem como de outros comportamentos), traços de personalidade e temperamento, ausência de um repertório de habilidades de enfrentamento adequadas e baixa autoeficácia( ${ }^{(24)}$.

Em revisão bibliográfica ${ }^{25)}$ apontou-se como principais causas do comportamento autolesivo o gerenciamento de angústia ou regulação de afeto para obter alívio de sentimentos como dor emocional, apagar estados cognitivos ou se acalmar; exercer influência interpessoal para mudar situações, requerer ajuda e amor ou para mostrar extensão da dor física; a punição relacionada a sentimentos bons; induzir estado dissociativo para não sentir nada ou sentir-se "dormente"; buscar sensações como excitação ou euforia relacionada a descarga de adrenalina produzida pelo corte; lidar com o risco de suicídio; manter ou explorar fronteiras a partir da criação de limites simbólicos entre o indivíduo e os outros; e expressar ou lidar com a própria sexualidade. Importante ressaltar que gerenciamento de angústia ou regulação de afeto foi o motivo preponderante na maioria dos estudos encontrados na revisão bibliográfica ${ }^{(25)}$.

\section{Conclusão}

O estudo permitiu identificar a composição e estrutura do grupo de automutilação da Rede Social Virtual Facebook a partir da identificação de suas características para operacionalização do grupo. Encontrado entre os participantes do grupo de automutilação no Facebook características identitárias de usuários adolescentes, faixa etária predominante para o comportamento autolesivo e que possui maior uso da Internet. O grupo de automutilação oferece apoio e compreensão a partir da cooperação, identificação e apoio mútuo entre os participantes contribuindo na prevenção do comportamento de automutilação, porém encontraramse também aspectos que evidenciam a vulnerabilidade desses adolescentes, como os transtornos alimentares, a prática de automutilação e isolamento social; bem como aspectos que fornecem vulnerabilidade, como influência por ídolos ou pelos pares, efeito contágio e gatilho, causados por fotos e/ou postagens.

Atualmente o fenômeno autolesivo tem atingindo grandes conjecturas e a internet tem sido utilizada como ferramenta de debate, busca de informações, grupos de apoio e/ou reforço de estigmas e comportamentos de risco. Estudos que desvelam o comportamento no ambiente on-line permitem olhar mais atual do fenômeno, o avanço de estratégias de identificação, prevenção e promoção de saúde e propicia o manejo por profissionais, sendo imprescindível conciliar o atendimento clínico com as redes sociais virtuais.

Em relação às limitações do estudo coloca-se a análise de postagens aleatórias, ou seja, a análise das postagens não teve associação com o participante que postou, sendo interessante acompanhar os depoimentos a partir do indivíduo e analisar as várias postagens que um mesmo participante pode postar. Compreende-se a necessidade de estudos que aprofundem e esclareçam a dinamicidade e funcionalidade dos conteúdos de grupos de automutilação em Rede Social Virtual, para melhor compreensão do fenômeno e concepção de práticas de prevenção e manejo do comportamento autolesivo.

Sugere-se estudos em diferentes contextos de vida dos adolescentes como comunidade, escola e serviço de saúde a fim de elaborar uma melhor compreensão do fenômeno e de diversas perspectivas.

\section{Referências}

1. Giusti JS. Automutilação: características clínicas e comparação com pacientes com transtorno obsessivo 
compulsivo. [Tese]. São Paulo: Universidade de São Paulo; 2012.

2. Mars B, Heron J, Biddle L, Donovan JL, Holley R, Piper $M$, et. al. Exposure to, and searching for, information about suicide and self-harm on the Internet: Prevalence and predictors in a population based cohort of young adults. $\mathrm{J}$ Affect Disord. 2015; (185): 239-45.

3. Nicholson S, Jenkins R, Meltzer H. Suicide thoughts, suicide attempts and self-harm. In: Adult Psychiatric Morbidity in England, 2007: Results of a household survey. [Internet]; 2009 [cited Ago 1 2016]. Available from: http:// www.hscic.gov.uk/pubs/psychiatricmorbidity07

4. Hawton K, Saunders KE, O'Connor RC. Self-harm and suicide in adolescents. Lancet. 2012;379:2373-82.

5. Secretaria de Comunicação Social (BR). Pesquisa brasileira de mídia 2015: hábitos de consumo de mídia pela população brasileira. [Internet] Brasília-DF; 2014. [Acesso 9 jan 2017]. Disponível em: http://www.secom. gov.br/atuacao/pesquisa/lista-de-pesquisas-quantitativase-qualitativas-de-contratos-atuais/pesquisa-brasileira-demidia-pbm-2015.pdf

6. Office for National Statistics. Internet Access Households and Individuals, 2012. [Internet] Great Britain; 2012 [cited Jul 25 2016]. Available from: http://www.ons. gov.uk/ons/rel/rdit2/Internet-access---households-andindividuals/2012/stb-Internet-access--households-andindividuals--2012.html

7. ComScore. Social networking explodes worldwide as sites increase their focus on cultural relevance. [Internet]; 2008 [cited Jul 25 2016]. Available from: http://www. comscore.com/Insights/Press-Releases/2008/08/SocialNetworking-World-Wide?cs_edgescape_cc=US

8. Baker TG, Lewis SP. Responses to online photographs of non-suicidal selfinjury: a thematic analysis. Arch Suicide Res. 2013;17: 223-35.

9. Wong PWC, Wa-Fu K, Yau RSP, Ma HHM, Law YW, Chang SS, et. al. Accessing Suicide-Related Information on the Internet: A Retrospective Observational Study of Search Behavior. J Med Internet Res. 2013 Jan 11;15(1):e3. doi: 10.2196/jmir.2181.

10. Bragazzi NL. A Google Trends-based approach for monitoring NSSI. Psychol Res Behav Manag. 2014;7(30):18.

11. O'Connor RC, Rasmussen S, Hawton K. Adolescent self-harm: a school based study in Northern Ireland. J Affect Disorders. 2014; 159:46-52.

12. Kirmayer LJ, Raikhel E, Rahimi S. Cultures of the Internet: Identity, community and mental health. [Transcult Psychiatry. 2013;50(2):165-91.

13. Baume $\mathrm{P}$, Cantor $\mathrm{CH}$, Rolfe A. Cybersuicide: the role of interactive suicide notes on the Internet. Crisis. 1997;18(2):73-9.
14. Bardin L. Análise de Conteúdo. Lisboa: Edições 70; 1977.

15. Reis DC, Almeida TAC, Miranda MM, Alves RH, Madeira AMF. Health vulnerabilities in adolescence: socioeconomic conditions, social networks, drugs and violence. Rev. Latino-Am. Enfermagem. 2013; 21(2):1-9. 16. Miller K, Wakefield JRH, Sani F. Identification with social groups is associated with mental health in adolescents: Evidence from a Scottish community sample. Psychiatry Res. 2015;228:340-6.

17. Ramos JS, Neto AFP, Bagrichevsky M. Cultura Identitária pró-anorexia: características de um estilo de vida em uma comunidade virtual. Interface - Comunic Saúde Educ. 2011;15: 447-60.

18. Olatunji BO, Cox R, Ebesutani C, Wall D. Self-harm history predicts resistance to inpatient treatment of body shape aversion in women with eating disorders: The role of negative affect. Psychiatry Res. 2015;65:37-46.

19. Oliveira AM, Machado M. A adolescência e a espetacularização da vida. Psicol. Soc. 2015; 27(3):529-36. 20. Giles DC, Maltby J. The role of media figures in adolescent development: relations between autonomy, attachment, and interest in celebrities. Perspect Individ Dif. 2004;36: 813-22.

21. Boon SD, Lomore CD. Admirer-celebrity relationships among young adults: explaining perceptions of celebrity influence on identity. Hum Commun Res. 2001;27:432-65.

22. Moreno MA, Ton A, Selkie E, Evans Y. Secret Society 123: Understanding the Language of Self-Harm on Instagram. J Adolesc Health. 2016;58:78-84.

23. Tomasello M. The ultra-social animal. Eur J Soc Psychol. 2014;44:187-94.

24. Donovan DM, Marlatt GA. Avaliação dos comportamentos dependentes. São Paulo: Roca; 2009.

25. Edmondson AJ, Brennan CA, House AO. Non-suicidal reasons for self-harm: A systematic review of self-reported accounts. J Affect Disord. 2016;191:109-17. 